\title{
Stability of barrier buckets with small or zero rf-barrier separations
}

\author{
K. Y. Ng* \\ Fermi National Accelerator Laboratory, P.O. Box 500, Batavia, Illinois 60510, USA
}

(Received 21 April 2005; published 7 June 2006)

\begin{abstract}
A barrier bucket with very small separation between the rf barriers (relative to the barrier widths) or even zero separation has its synchrotron tune decreasing rather slowly from a large value towards the boundary of the bucket. As a result, a large area at the bucket edges can become unstable under the modulation of rf voltage and/or rf phase. In addition, chaotic regions may form near the bucket center and extend outward under increasing modulation. Application is made to those barrier buckets once used in the process of momentum mining at the Fermilab Recycler Ring.
\end{abstract}

DOI: $10.1103 /$ PhysRevSTAB.9.064001

PACS numbers: 02.90.+p, 05.45.Pq, 29.20.Dh

\section{INTRODUCTION}

In an earlier scenario of momentum mining at the Fermilab Recycler Ring, a barrier bucket with zero distance between the two barrier waves is opened to store the unmined particles. With the barrier width $T_{1}=1.27 \mu \mathrm{s}$ and height $V_{0}=2 \mathrm{kV}$, the maximum half-energy height of the bucket is

$$
\Delta E_{\mathrm{pk}}=\sqrt{\frac{e V_{0} T_{1}}{T_{0}} \frac{2 \beta^{2} E_{0}}{|\eta|}}=21.77 \mathrm{MeV},
$$

and the bucket area is

$$
\mathcal{B}=\frac{8}{3} T_{1} \Delta E_{\mathrm{pk}}=73.72 \mathrm{eV} \mathrm{s},
$$

where the nominal beam energy is $E_{0}=8.938 \mathrm{GeV}$, the nominal velocity is $v=\beta c$ with $c$ being the velocity of light, the revolution period is $T_{0}=11.13 \mu \mathrm{s}$, and the slip factor is $\eta=-0.008812$. All the expressions quoted in the section will be derived in Sec. II.

The synchrotron frequency at the center of the bucket is infinite, and at the edge assumes the value [1]

$$
\nu_{s, \min }=\frac{e V_{0}}{4 \Delta E_{\mathrm{pk}}}=2.297 \times 10^{-5} \text {. }
$$

For a particle with maximum energy offset $\widehat{\Delta E}<\Delta E_{\mathrm{pk}}$, the synchrotron tune decreases with $\widehat{\Delta E}$ hyperbolically according to

$$
\nu_{s}(\widehat{\Delta E})=\frac{e V_{0}}{4 \widehat{\Delta E}}=\nu_{s, \min } \frac{\Delta E_{\mathrm{pk}}}{\widehat{\Delta E}},
$$

and is plotted in Fig. 1. Because of the rather slowly decreasing synchrotron tune towards the edge of the bucket, fixed points of parametric resonances will exhibit themselves rather far away from the edge of the bucket in the presence of voltage and/or rf phase modulation. The implication is that particles well inside the bucket can be

*Electronic address: ng@fnal.gov driven to the edges, resulting in bunch area increase and possibly beam loss.

For a sinusoidal rf bucket, the very edge of the bucket under modulation is usually chaotic because of overlapping resonances. As the modulation strength or tune increases, the chaotic region spreads inward. As a result, there is always an area close to the bucket center that is stable. For a barrier bucket with zero or very short barrier separation, the situation reverses. Chaotic region, if present, starts from the central region of the bucket instead. As the modulation strength or modulation tune increases, the chaotic region spreads outward until eventually the whole bucket becomes chaotic and thus unstable. This is illustrated schematically in Fig. 2.

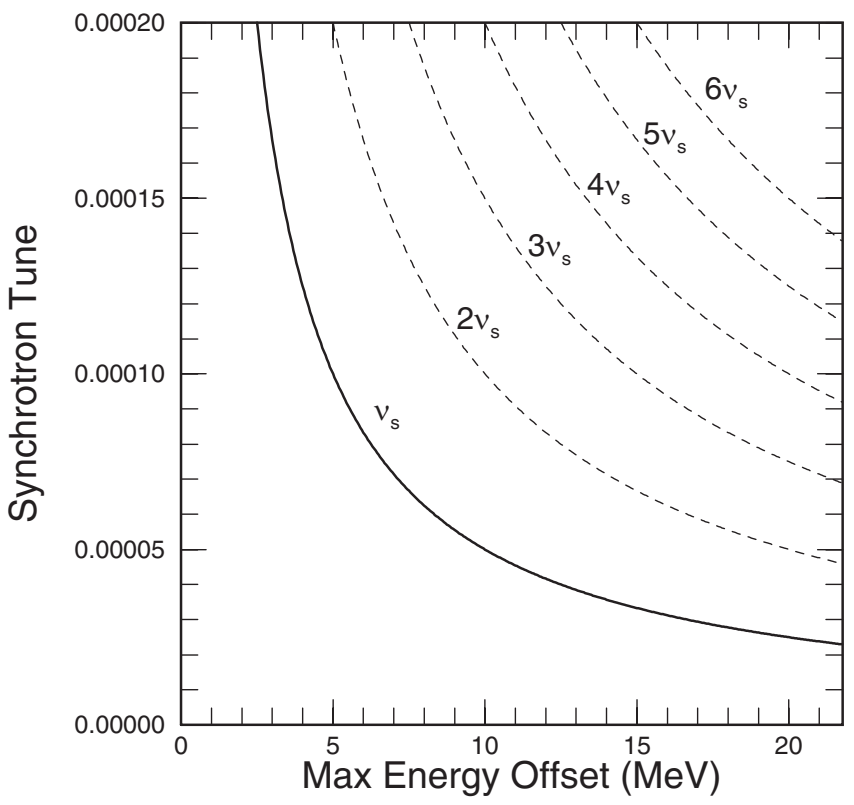

FIG. 1. Synchrotron tune as functions of energy offset, when the barrier separation is $T_{2}=0$, the barrier width $T_{1}=1.27 \mu \mathrm{s}$, and the barrier voltage $V_{0}=2 \mathrm{kV}$. Also shown in the plots are higher synchrotron harmonics. 

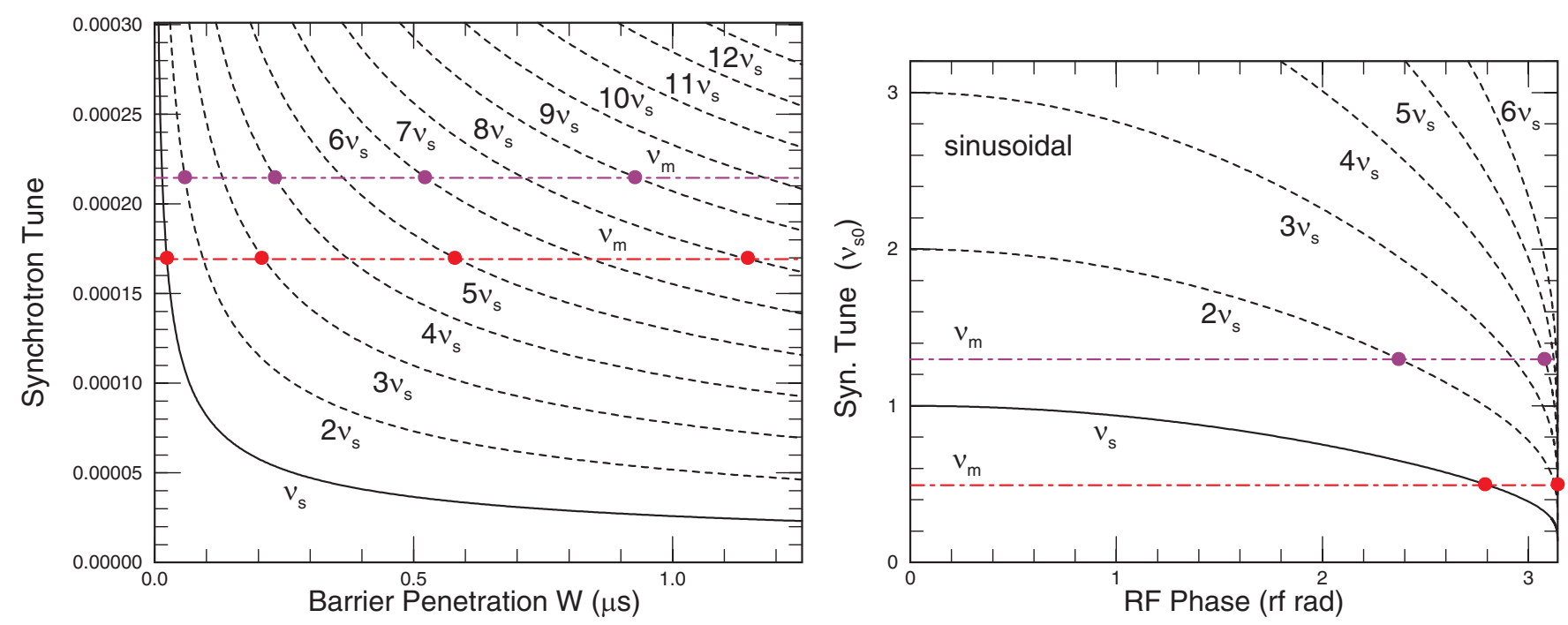

FIG. 2. (Color) Synchrotron tune and harmonics versus barrier penetration showing resonance chain (dot-dashes) starts near the center of the bucket and spreads towards the edge in a barrier bucket with zero barrier separation (left). The reverse is true for the sinusoidal rf bucket on the right.

\section{BEAM DYNAMICS OF UNPERTURBED BARRIER SYSTEM}

Notice that the arrival time $\tau$ of a beam particle at a certain location of the accelerator ring ahead of some onenergy particle is in the opposite direction of the rf phase. In order to avoid a negative argument in the rf wave, here we define $\tau$ instead as the arrival time lagging behind some on-energy particle. We choose $\tau$ and the energy offset $\Delta E$ as the canonical variables for the description of the beam particle. The independent variable is $\theta$, the longitudinal azimuthal angle along the designed orbit, and it advances by $2 \pi$ in a revolution turn. In the absence of voltage or phase modulation, the equations of motion are [3]

$$
\begin{gathered}
\frac{d \tau}{d \theta}=\frac{\eta \Delta E}{\omega_{0} \beta^{2} E_{0}}, \\
\frac{d \Delta E}{d \theta}=\frac{e V_{0} T_{1}}{2 \pi} \frac{\partial f_{0}}{\partial \tau}=\frac{e V_{0}}{2 \pi} f_{1}\left(\tau, T_{1}\right),
\end{gathered}
$$

where the reduced barrier voltage wave $f_{1}(\tau)$ and the reduced barrier potential $f_{0}(\tau)$ are depicted in Fig. 3 and can be written explicitly as

$$
\begin{aligned}
f_{1}\left(\tau, T_{1}\right) & =T_{1} \frac{\partial f_{0}\left(\tau, T_{1}\right)}{\partial \tau} \\
& =-\theta\left(\tau+T_{1}\right)+2 \theta(\tau)-\theta\left(\tau-T_{1}\right), \\
f_{0}\left(\tau, T_{1}\right)= & \frac{1}{T_{1}}\left[-\left(\tau+T_{1}\right) \theta\left(\tau+T_{1}\right)+2 \tau \theta(\tau)\right. \\
& \left.-\left(\tau-T_{1}\right) \theta\left(\tau-T_{1}\right)\right]+1 .
\end{aligned}
$$

We have normalized the rf potential in such a way that it vanishes at the center of the barrier bucket. The
Hamiltonian is given by

$$
H_{0}=\frac{\eta(\Delta E)^{2}}{2 \omega_{0} \beta^{2} E_{0}}-\frac{e V_{0} T_{1}}{2 \pi} f_{0}\left(\tau, T_{1}\right) .
$$

The Recycler Ring operates below transition with $\eta<0$. The particles perform stable oscillations inside the barrier bucket in the anticlockwise direction. It is unfortunate that the new definition of $\tau$ makes the Hamiltonian assume negative values (and so will the action introduced below). For a particle with maximum energy offset $\widehat{\Delta E}$, the Hamiltonian value is

$$
H_{0}=-\frac{|\eta|(\widehat{\Delta E})^{2}}{2 \omega_{0} \beta^{2} E_{0}}=-\frac{e V_{0} W}{2 \pi},
$$

where the parameter $W$ has the dimension of time and represents the largest penetration into the barrier wave.

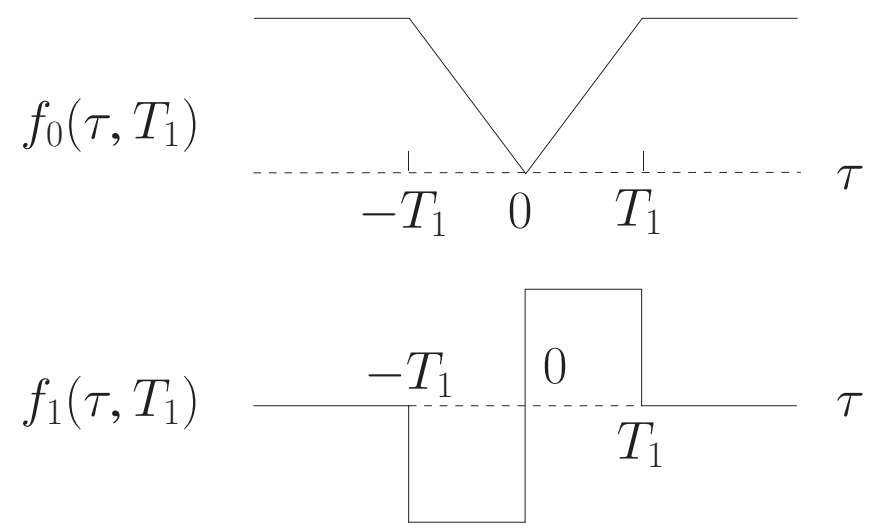

FIG. 3. Rf potential $f_{0}\left(\tau, T_{1}\right)$ and $\mathrm{rf}$ force $f_{1}\left(\tau, T_{1}\right)=$ $T_{1} \partial f_{0}\left(\tau, T_{1}\right) / \partial \tau$. 
Thus $W=0$ corresponds to an on-momentum particle and $W=T_{1}$ is associated with particles on the bucket boundary. For the particle to be stable inside the barrier bucket, we require $W \leq T_{1}$. One can then solve for the energy offset for any arrival time $\tau$ according to

$$
\Delta E= \pm \sqrt{\frac{2 \beta^{2} E_{0} e V_{0}}{|\eta| T_{0}}} \sqrt{W-T_{1} f_{0}\left(\tau, T_{1}\right)} .
$$

Let us go to the action-angle variables. The unperturbed Hamiltonian $H_{0}$ is a constant of motion. The action torus is given by

$$
\begin{aligned}
J & =\frac{1}{2 \pi} \oint \Delta E d \tau \\
& =\frac{1}{2 \pi} \sqrt{\frac{\omega_{0} \beta^{2} E_{0} e V_{0}}{\pi|\eta|}} \oint\left[ \pm \sqrt{W-T_{1} f_{0}\left(\tau, T_{1}\right)}\right] d \tau,
\end{aligned}
$$

where the contour of integration is to follow the particle trajectory in the anticlockwise direction. The action for a particle torus inside the bucket is found to be

$$
J=-\frac{1}{2 \pi}\left[\frac{8}{3} W \widehat{\Delta E}\right]=-\frac{1}{2 \pi} \sqrt{\frac{\omega_{0} \beta^{2} E_{0} e V_{0}}{\pi|\eta|}} \frac{8}{3} W^{3 / 2}
$$

The particle trajectory represents the enclosure of an area

$$
\mathcal{A}=\frac{8}{3} W \widehat{\Delta E},
$$

and the largest possible stable area enclosed is the bucket area when $W=T_{1}$, as given by Eq. (1.2).

Canonical transformation from the phase space coordinates $(\tau, \Delta E)$ to the action-angle variables can be achieved via the generating function

$$
F_{2}(J, \tau)=\int_{0}^{\tau} \Delta E d \tau
$$

where $\tau$ represents the penetration of the torus into the $\mathrm{rf}$ voltage barrier. The angle variable is given by

$$
\begin{aligned}
\psi & =\frac{\partial F_{2}}{\partial J}=\frac{d W}{d J} \int_{0}^{\tau} \frac{\partial \Delta E}{\partial W} d \tau \\
& =-\frac{\pi}{4 \sqrt{W}} \int_{0}^{\tau} \frac{d \tau}{ \pm \sqrt{W-T_{1} f_{0}}},
\end{aligned}
$$

where the \pm sign corresponds to the situation when $\Delta E \gtrless$ 0 . Since $f_{0}\left(\tau, T_{1}\right)$ has definite parity with respect to $\tau$, we choose $\psi=0$ at $\tau=0$ and $\Delta E>0$. Thus it is negative in the first and fourth quadrants, positive in the second and third quadrants. Starting from $\tau=0$ and $\Delta E<0$ and moving anticlockwise, the angle variable in one synchrotron oscillation assumes the value

$$
\psi= \begin{cases}-\frac{\pi}{2}\left(1+\sqrt{1-\frac{\tau}{W}}\right) & 0 \leq \tau \leq W, \Delta E<0 \\ -\frac{\pi}{2}\left(1-\sqrt{1-\frac{\tau}{W}}\right) & 0 \leq \tau \leq W, \Delta E>0 \\ +\frac{\pi}{2}\left(1-\sqrt{1+\frac{\tau}{W}}\right) & -W \leq \tau \leq 0, \Delta E>0 \\ +\frac{\pi}{2}\left(1+\sqrt{1+\frac{\tau}{W}}\right) & -W \leq \tau \leq 0, \Delta E<0\end{cases}
$$

In short, canonical transformation is accomplished as follows. Given the position $\tau$ and energy offset $\Delta E$ of a particle, the maximum barrier penetration $W$ can be computed via Eq. (2.7) and therefore the action $J$ is known. The angle variable $\psi$ is obtained through Eq. (2.13).

The synchrotron tune can be derived from the Hamilton equation via

$$
\frac{d \psi}{d \theta}=\frac{\partial H_{0}}{\partial J} .
$$

Since $d \psi / d \theta=\nu_{s}$, we obtain the synchrotron tune of a particle with trajectory penetrating the barrier by $W$

$$
\nu_{s}(W)=\sqrt{\frac{|\eta| e V_{0} T_{0}}{32 \beta^{2} E_{0} W}}=\frac{e V_{0}}{4 \widehat{\Delta E}}=\nu_{s, \min } \sqrt{\frac{T_{1}}{W}} .
$$

\section{VOLTAGE MODULATION}

To introduce voltage modulation, we make the substitution

$$
V_{0} \rightarrow V_{0}\left(1+a \cos \nu_{m} \theta\right)
$$

where $\nu_{m}$ is the modulation tune and $a V_{0}$ is the modulation voltage. We also call $a$ the modulation amplitude. The Hamiltonian receives a perturbative term

$$
\Delta H=-\frac{e V_{0} T_{1}}{2 \pi} f_{0}\left(\tau, T_{1}\right) a \cos \nu_{m} \theta .
$$

We expand $f_{0}\left(\tau, T_{1}\right)$ in action-angle variables,

$$
f_{0}\left(\tau, T_{1}\right)=\sum_{m=-\infty}^{\infty} F_{m}(J) e^{i m \psi} .
$$

Since $f_{0}$ is an even function of $\tau, F_{m}(J)=F_{-m}(J)$ and only even synchrotron harmonics contribute, or [4]

$$
F_{m}(J)= \begin{cases}0 & m \text { odd }, \\ \frac{2 W}{3 T_{1}} & m=0, \\ -\frac{8 W}{m^{2} \pi^{2} T_{1}} & m \text { even but } \neq 0,\end{cases}
$$

The resonance strength functions satisfy the sum rule

$$
\sum_{m=-\infty}^{\infty}\left|F_{m}\right|^{2}=\frac{4 W^{2}}{45 T_{1}^{2}}+\frac{4 W^{2}}{9 T_{1}^{2}},
$$

where the last term is the contribution of the $m=0$ component. The effect of rf voltage modulation is concentrated 


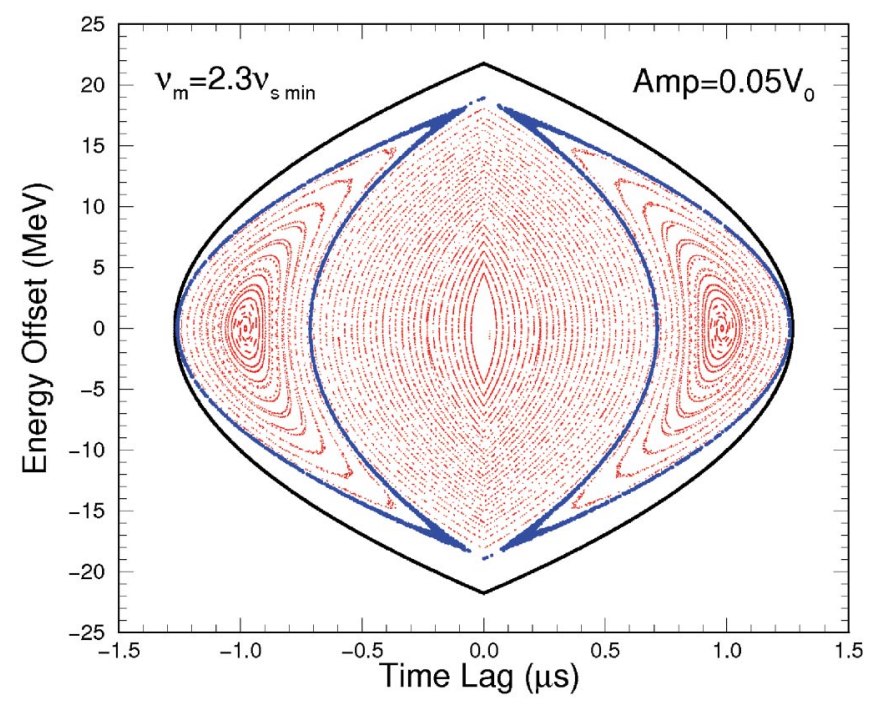

FIG. 4. (Color) Poincaré section showing the excitation of the $2: 1$ resonance by rf voltage modulation at $\mu_{m}=2.3 \nu_{s, \text { min }}$ and modulation amplitude $a V_{0}=0.05 V_{0}$. The separatrices shown just touch the barrier bucket boundary, indicating that particles situation near the inner branches of the separatrices will be lost. The simulation involves initially 100 randomly but uniformly distributed particles and has been tracked for $5 \times 10^{6}$ revolution turns $(55.7 \mathrm{~s})$.

at low synchrotron harmonics because of the $m^{-2}$ dependency.

The perturbation term in the Hamiltonian can be rewritten as

$$
\begin{aligned}
\Delta H= & -\frac{a e V_{0} W}{3 \pi} \cos \nu_{m} \theta+\sum_{n=1}^{\infty} \frac{a e V_{0} W}{n^{2} \pi^{3}}\left[\cos \left(2 n \psi+\nu_{m} \theta\right)\right. \\
& \left.+\cos \left(2 n \psi-\nu_{m} \theta\right)\right],
\end{aligned}
$$

which exhibits all the possible parametric resonances. We see that whenever the modulation frequency is an even harmonic of the synchrotron frequency, the rf voltage modulation can perturb the particle motion severely. The most important modulation of concern is near the boundary of the bucket where the synchrotron tune assumes the minimum value of $\nu_{s, \min }$. Thus when $\nu_{m}=2 \nu_{s, \min }$, particles near the boundary of the bucket are subject to the 2:1 parametric resonance and beam loss will occur.

Simulations have been performed to study the effects of voltage modulation. An example is shown in Fig. 4, where we track 100 randomly distributed particles according to a uniform distribution for $5 \times 10^{6}$ turns $(55.7 \mathrm{~s})$. The peak rf voltage $V_{0}$ is modulated by the fractional amplitude $a=$ 0.05 at the modulation tune of $\nu_{m}=2.30 \nu_{s, \min }$. The Poincaré section is recorded every modulation period or $1 / \nu_{m} \approx 18928$ revolution turns. We see in the plot the $2: 1$ resonance. Besides the center of the phase space, there are two other stable fixed points at $\Delta E=0$ and two unstable fixed points at $\tau=0$, which make up the 2:1 resonant island chain. Their exact locations can be computed by picking out only the relevant resonance from the Hamiltonian in Eqs. (2.6) and (3.6). Let us concentrate on the $2 n: 1$ resonance with $n=1,2,3, \cdots$. Thus

$$
H(J, \psi)=-p(-J)^{2 / 3}\left[1-\frac{2 a}{n^{2} \pi^{2}} \cos \left(2 n \psi-\nu_{m} \theta\right)\right],
$$

where

$$
p=\frac{e V_{0}}{2 \pi}\left(\frac{3 \pi}{4}\right)^{2 / 3}\left(\frac{T_{0}|\eta|}{2 \beta^{2} E_{0} e V_{0}}\right)^{1 / 3},
$$

with both $p>0$ and $-J>0$. Next the generating function

$$
F_{2}(\psi, \bar{J})=\left(\psi-\frac{\nu_{m}}{2 n} \theta\right) \bar{J}
$$

is used to transform the Hamiltonian to a frame rotating with the modulation. The result is

$$
H(J, \psi)=-p(-J)^{2 / 3}\left[1-\frac{2 a}{n^{2} \pi^{2}} \cos 2 n \psi\right]-\frac{\nu_{m}}{2 n} J,
$$

where the bars on $\psi$ and $J$ have been removed to ease writing. The fixed points are given by

$$
\begin{aligned}
& \frac{\partial H}{\partial \psi}=-2 n p(-J)^{2 / 3} \frac{2 a}{n^{2} \pi^{2}} \sin 2 n \psi=0, \\
& \frac{\partial H}{\partial J}=\frac{2 p}{3(-J)^{1 / 3}}\left(1-\frac{2 a}{n^{2} \pi^{2}} \cos 2 n \psi\right)-\frac{\nu_{m}}{2 n}=0 .
\end{aligned}
$$

Recall that stable fixed points correspond to $\partial^{2} H / \partial \psi^{2}$ and $\partial^{2} H / \partial J^{2}$ having the same sign while unstable fixed points correspond to $\partial^{2} H / \partial \psi^{2}$ and $\partial^{2} H / \partial J^{2}$ having opposite signs. We obtain the fixed points:

$$
\begin{gathered}
\text { Unstable: }(-J)^{1 / 3}=\frac{4 n p}{3 \nu_{m}}\left(1-\frac{2 a}{n^{2} \pi^{2}}\right), \psi=\frac{\pi n^{\prime}}{2 n}, n^{\prime}=0, \pm 2, \pm 4, \ldots, 2 n, \\
\text { Stable: }(-J)^{1 / 3}=\frac{4 n p}{3 \nu_{m}}\left(1+\frac{2 a}{n^{2} \pi^{2}}\right), \psi=\frac{\pi n^{\prime}}{2 n}, n^{\prime}= \pm 1, \pm 3, \ldots, \pm(2 n-1) .
\end{gathered}
$$

Thus for the $2 n: 1$ resonance, we have $n+1$ stable fixed points and unstable fixed points, or a circular chain of $n+1$ islands. We can conclude that the fixed points at $\psi=0$ and $\pi$ are always unstable. At $\psi= \pm \pi / 2$, the fixed points are stable when $n$ is odd and unstable when $n$ is even. Notice that in the convention of Eq. (2.13), $\psi=0, \pi$ represent the positive and negative $\Delta E$ axes, while $\psi= \pm \pi / 2$ represent the negative and positive $\tau$ axes. We are especially interested in 
the fixed points on the $\tau$ and $\Delta E$ axes. Thus, on the $\Delta E$ axis, the fixed points are located at

$$
\Delta E= \pm \frac{2 n \nu_{s, \min }}{\nu_{m}}\left(1-\frac{2 a}{n^{2} \pi^{2}}\right) \Delta E_{\mathrm{pk}}, \quad \tau=0,
$$

and are unstable, while on the $\tau$ axis, the fixed points are at

$$
\tau= \pm\left(\frac{2 n \nu_{s, \min }}{\nu_{m}}\right)^{2}\left[1-(-1)^{n} \frac{2 a}{n^{2} \pi^{2}}\right]^{2} T_{1}, \quad \Delta E=0,
$$

and are stable/unstable when $n$ is odd/even. In deriving the above, Eqs. (2.6) and (2.9) have been used. Since the modulation amplitude is usually small, the factors $[1 \pm$ $\left.2 a /(n \pi)^{2}\right]$ can be neglected (here in the simulation with $a=0.05$ for the $2: 1$ resonance, $2 a / \pi^{2}=0.01$ ). In this approximation, the fixed points for the 2:1 resonance can be determined much easier. For example, the time lags of the stable fixed points can be obtained through Eq. (2.15) by equating $\nu_{m}$ to twice the synchrotron tune of the particle:

$$
\tau= \pm\left(\frac{2 \nu_{s, \min }}{\nu_{m}}\right)^{2} T_{1}=0.96 \mu \mathrm{s}
$$

Graphically, they can be solved by plotting $2 \nu_{s}$ as a function of $W$ and drawing a line $\nu_{m}=2.3 \nu_{s, \min }$. Where the line and the curve meet gives the stable fixed point. The two unstable fixed points at $\tau=0$ can also be determined in the same way by equating the modulation tune to twice the synchrotron tune, or

$$
\frac{\Delta E}{E_{\mathrm{pk}}}= \pm \frac{2 \nu_{s, \min }}{\nu_{m}}= \pm 0.87
$$

Since the separatrices touch the outside edge of the barrier bucket, particles residing near the separatrices will be driven to the edge of the bucket and will possibly be lost eventually. We would like to compute the widths of the $2 n: 1$ resonant islands by determining the intercepts of the separatrices with the time-lag axis. From Eq. (3.10), the separatrices are given by

$$
\begin{aligned}
- & p(-J)^{2 / 3}\left(1-\frac{2 a}{n^{2} \pi^{2}} \cos 2 n \psi\right)-\frac{\nu_{m}}{2 n} J \\
= & -\frac{16 n^{2} p^{3}}{27 \nu_{m}^{2}}\left(1-\frac{2 a}{n^{2} \pi^{2}}\right)^{3}
\end{aligned}
$$

where the right side is the Hamiltonian value evaluated at any of the unstable fixed points, for example, at $\psi=0$. To derive the required intercepts, we set $\psi=\pi / 2$. Introducing the parameters

$$
x=\frac{3 \nu_{m}(-J)^{1 / 3}}{4 n p(1-A)}, \quad \alpha=\sqrt{\frac{1-A}{1-(-1)^{n} A}}, \quad A=\frac{2 a}{n^{2} \pi^{2}},
$$

the intercepts are now given by the solutions of the cubic equation

$$
2 x^{3}-\frac{3}{\alpha^{2}} x^{2}+1=0,
$$

which can be rewritten as

$$
3\left(\frac{\alpha}{2 x}\right)-4\left(\frac{\alpha}{2 x}\right)^{3}=\alpha^{3} .
$$

The solution can now be easily written down by identifying $\alpha /(2 x)$ with $\sin \phi$, or

$$
x=\frac{\alpha}{2 \sin \phi} \quad \text { with } \phi=\left\{\begin{array}{l}
\frac{1}{3} \sin ^{-1} \alpha^{3} \\
\frac{1}{3} \sin ^{-1} \alpha^{3}+\frac{2 \pi}{3} \\
\frac{1}{3} \sin ^{-1} \alpha^{3}+\frac{4 \pi}{3} .
\end{array}\right.
$$

The third solution gives $x \sim(-J)^{1 / 3}<0$, which is considered unphysical. For even $n, \alpha=1$ and both the first and second solutions give $x=1$, or

$$
(-J)^{1 / 3}=\frac{4 n p}{3 \nu_{m}}
$$

We get only one intercept here because the separatrices intercept the positive $\tau$ axis at an unstable fixed point when $n$ is even. When $n$ is odd, however, a stable fixed point of the island chain meets the positive $\tau$ axis and the first two solutions of Eq. (3.21) represent the intercepts of the edges of an island at the positive $\tau$ axis. When the modulation amplitude is small, i.e., $a$ or $A \ll 1$, an approximate solution of the intercepts is desired. Unfortunately, $\sin ^{-1} \alpha^{3}$ cannot be expanded as a power series in $A$ because the derivative diverges at $A=0$. However, we can expand it in terms of $\sqrt{A}$, resulting in the first 2 solutions:

$$
x=1 \pm \sqrt{2 A}+\frac{4}{3} A+\mathcal{O}\left(A^{3 / 2}\right) .
$$

Recalling that $\tau \propto(-J)^{2 / 3}$ when $\Delta E=0$, the two intercepts are

$$
(-J)^{2 / 3}=\left(\frac{4 n p}{3 \nu_{m}}\right)^{2}\left[1 \pm 2 \sqrt{2 A}+\frac{8}{3} A+\mathcal{O}\left(A^{3 / 2}\right)\right]
$$

or the half-widths of the resonant islands are

$$
\delta(-J)^{2 / 3}=2 \sqrt{2 A}\left(\frac{4 n p}{3 \nu_{m}}\right)^{2},
$$

which is accurate up to $\mathcal{O}(A)$.

Coming back to our simulation of the 2:1 resonance, the first and second solutions correspond to $\tau=1.26 \mu \mathrm{s}$ and $0.712 \mu \mathrm{s}$, which are, respectively, the outer and inner intercepts. Thus to avoid bunch lengthening and beam loss, particles injected into this bucket should not exceed $|\tau| \approx 0.71 \mu \mathrm{s}$ and $|\Delta E| \approx 0.87 E_{\mathrm{pk}}$. If we keep the modulation amplitude fixed and lower the modulation tune, the outer separatrices will exceed the width of the bucket, implying that particles near the inner separatrices will be driven outside the barrier bucket resulting in beam loss. 
Thus $|\tau| \lesssim 0.71 \mu$ s does set the limit of beam population to prevent beam loss at the modulation amplitude of $a=$ 0.05 regardless of the modulation tune. It is important to point out that since the Hamiltonian in Eq. (3.10) contains only one parametric resonance, namely, the $2 n: 1$ resonance, the separatrix forms a boundary between the resonant islands and the rest of the longitudinal phase space. For the actual Hamiltonian which contains all other parametric resonances, the separatrix is no longer a thin curve and the vicinity close to the separatrix is chaotic, implying that particles near the separatrix can jump across the separatrix from one region to another.

For large modulation tune $\nu_{m} / \nu_{s, \min } \gg 1$, many parametric resonances will be excited, because the $\nu_{m}$ intersects all the $2 n \nu_{s}$ curves for $n=1,2, \cdots$ up to $\nu_{m} /\left(2 \nu_{s, \min }\right)$ in Fig. 1 in view of the $\nu_{s}(W)=$ $\nu_{s, \min } \sqrt{T_{1} / W}$ behavior of the synchrotron tune. It is important to determine the separation of neighboring chains of parametric resonant islands. If they overlap one another, a chaotic sea will be formed.

An example is shown in Fig. 5, where the modulation voltage is $a V_{0}=0.05 V_{0}$ and modulation tune $\nu_{m}=$ $29.08 \nu_{s, \text { min }}$ which corresponds to a modulation frequency of $60 \mathrm{~Hz}$. Sixty randomly distributed particles are used and the tracking has been for $5 \times 10^{6}$ turns. We see a ring of 28 resonance islands just inside the edge of the bucket. Going inwards are rings of $26,24, \cdots$ islands. It is evident that the rings of islands are well separated without overlapping and there is no large area of chaos near the center of the bucket. The absence of a chaotic sea can be understood mathe-

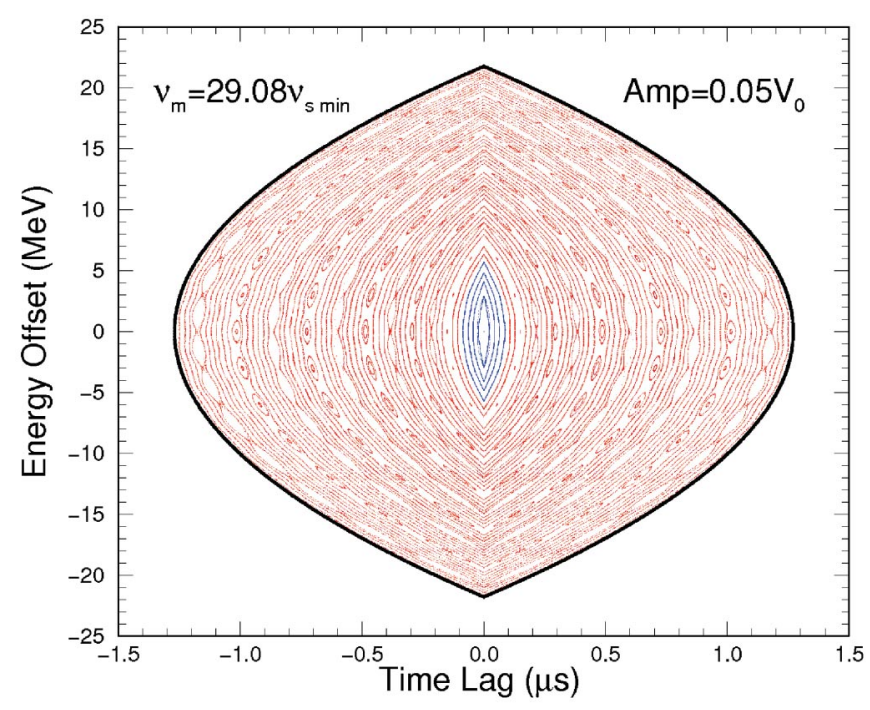

FIG. 5. (Color) Poincaré section showing the voltage modulation with amplitude $a V_{0}=0.05 V_{0}$ and modulation tune $\nu_{m}=$ $29.08 \nu_{s, \min }$ (or modulation frequency $60 \mathrm{~Hz}$ ). The simulations was performed with 60 equally spaced particles on the positive time-advance axis and tracking for $5 \times 10^{6}$ turns. Chains of resonant islands are seen well separated without any chaotic region near the center of the bucket. matically. The separation between fixed points of the $2 n: 1$ and $2(n+1): 1$ resonances is given by

$$
\Delta(-J)^{2 / 3}=\left(\frac{4 p}{3 \nu_{m}}\right)^{2}\left[(n+1)^{2}-n^{2}\right]=\left(\frac{4 p}{3 \nu_{m}}\right)^{2}(2 n+1),
$$

valid when $a \ll n^{2} \pi^{2} / 2$ which should be true always, while the half-width of the resonant island is given by Eq. (3.25). Thus the condition for nonoverlapping islands implies [5] $\delta(-J)^{2 / 3}<\Delta(-J)^{2 / 3}$, or, using Eq. (3.18),

$$
|a|<\left[\left(1+\frac{1}{2 n}\right) \frac{\pi}{2}\right]^{2}
$$

This concludes that there will not be overlapping of resonant island chains when the modulation amplitude $|a|<1$. It is interesting to observe that this criterion of no overlapping of adjacent island chains is independent of the modulation tune. It depends only on the parametric numbering of the island chain. When overlapping occurs, the chaotic region starts from the central part of the bucket and extends outward as the modulation amplitude increases. We do see in simulation that the central region becomes chaotic when $|a| \geq 1.5$.

To estimate the tolerance of the rf voltage modulation, we calculate the maximum stable bunch area of the $\mathrm{rf}$ system. We randomly and uniformly populate 5000 particles inside the bucket area and track the beam for more than 100 synchrotron oscillations (with reference to $\nu_{s, \min }$ ). The fractional stable area in units of the bucket area is defined as the ratio between the number of survival particles and the number of initial particles. The result is depicted in Fig. 6 as functions of modulation tune. The traces from the top downwards correspond to fractional modulation amplitude $a=0.001,0.005,0.01,0.02,0.03$, $0.04,0.05,0.06,0.07,0.08,0.09$, and 0.1 . The parametric resonances corresponding to even synchrotron harmonics are evident. Aside from the resonances, it appears that the bucket cannot be filled to more than $95 \%$ without encountering beam loss.

\section{RF PHASE MODULATION}

To introduce rf phase modulation, we make the substitution

$$
\tau \rightarrow \tau+T_{1} a \cos \nu_{m} \theta
$$

where $\nu_{m}$ is the modulation tune. The perturbation term in the Hamiltonian becomes

$$
\Delta H=-\frac{e V_{0} T_{1}}{2 \pi} f_{1}\left(\tau, T_{1}\right) a \cos \nu_{m} \theta,
$$

where the function $f_{1}\left(\tau, T_{1}\right)$ is the reduced barrier voltage profile defined in Eq. (2.3) and depicted in Fig. 3. We expand $f_{1}$ in action-angle variables, 


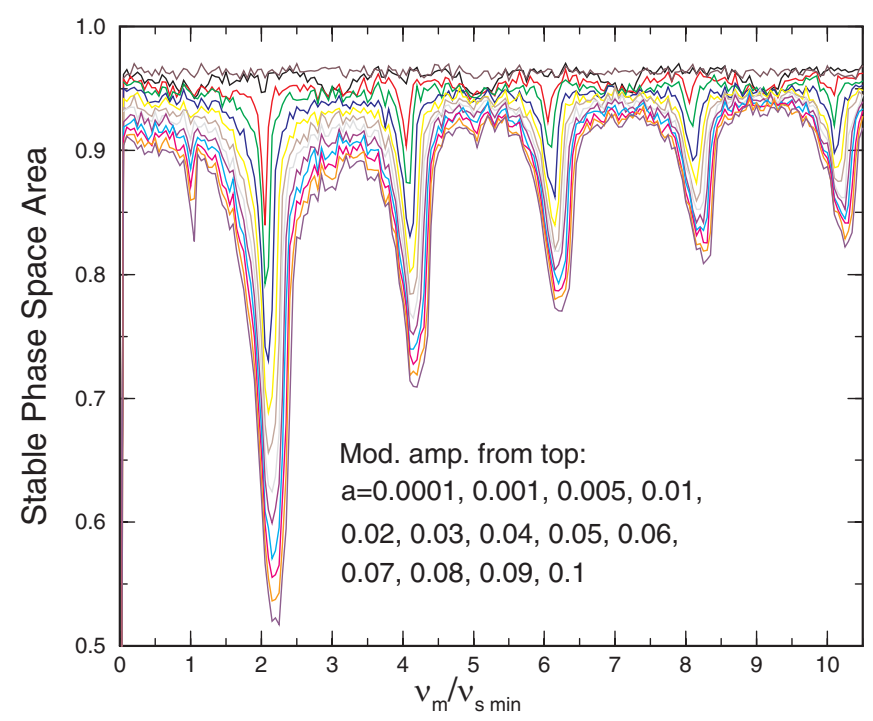

FIG. 6. (Color) Fractional stable bunch area in ratio to the bucket area as functions of voltage modulation tune, for fractional modulation amplitudes, from top to bottom, $a=0.001$, $0.005,0.01,0.02,0.03,0.04,0.05,0.06,0.07,0.08,0.09,0.1$. Large unstable areas are seen when modulation tune $\nu_{m}$ slightly larger than $n \nu_{s, \min }$ with $n=2,4,6,8, \cdots$, a reflection of the resonances excited. Among them, the $2: 1$ resonance $(n=2)$ is the most devastating.

$$
f_{1}\left(\tau, T_{1}\right)=\sum_{m=-\infty}^{\infty} G_{m}(J) e^{i m \psi}
$$

Since $f_{1}$ is an odd function of $\tau$, only odd synchrotron harmonics contribute and $G_{m}(J)=G_{-m}^{*}(J)$, or

$$
G_{m}(J)= \begin{cases}0 & m \text { even } \\ -\frac{2 i}{m \pi} & m \text { odd }\end{cases}
$$

The resonance strength functions satisfy the sum rule

$$
\sum_{-\infty}^{\infty}\left|G_{m}(J)\right|^{2}=1
$$

The perturbation term in the Hamiltonian becomes

$$
\Delta H=-\sum_{m=1,3, \cdots} \frac{a e V_{0} T_{1}}{m \pi^{2}}\left[\sin \left(m \psi+\nu_{m} \theta\right)+\sin \left(m \psi-\nu_{m} \theta\right)\right] .
$$

We see that whenever the modulation frequency is an odd harmonic of the synchrotron frequency, the rf phase modulation can coherently perturb the particle motion. The effect of rf phase modulation will be stronger than the effect of voltage modulation, because of the $m^{-1}$ dependency of the resonance strength functions and the larger value of the sum of their squares. The most important modulation of concern is near the boundary of the bucket where the synchrotron tune assumes the minimum value of $\nu_{s, \min }$. Thus when $\nu_{m}=\nu_{s, \min }$, particles near the boundary of the bucket are subject to the 1:1 parametric resonance and beam loss will occur.

Simulations have been performed to study the effects of rf phase modulation. An example is shown in Fig. 7. The rf phase is modulated by the amplitude $a T_{1}=0.02 T_{1}$ at the modulation tune of $\nu_{m}=1.195 \nu_{s, \min }$. The Poincaré section is recorded every modulation period or $1 / \nu_{m} \approx$ 36432 revolution turns. We see in the plot the $1: 1$ resonance, which is not left-right symmetric. The central stable region has its stable fixed point shifted to the right. There exists also a set of bifurcated stable and unstable fixed points at the time-advance axis. Their positions can be determined by retaining only the $m: 1$ resonant term with $m=1,3,5, \cdots$ in the Hamiltonian in the frame rotating with the modulation:

$$
H(J, \psi)=-p(-J)^{2 / 3}-\frac{\nu_{m}}{m} J-\frac{a e V_{0} T_{1}}{m \pi^{2}} \sin m \psi .
$$

Since

$$
\frac{\partial H}{\partial \psi}=-\frac{a e V_{0} T_{1}}{\pi^{2}} \cos m \psi, \quad \frac{\partial H}{\partial J}=\frac{2 p}{3(-J)^{1 / 3}}-\frac{\nu_{m}}{m},
$$

the fixed points are at

$$
(-J)^{1 / 3}=\frac{2 m p}{3 \nu_{m}} \quad \text { and } \quad \psi= \pm \frac{\pi m^{\prime}}{2 m},
$$

with $m^{\prime}=1,3, \ldots, 2 m-1$. It is easy to see that the chain

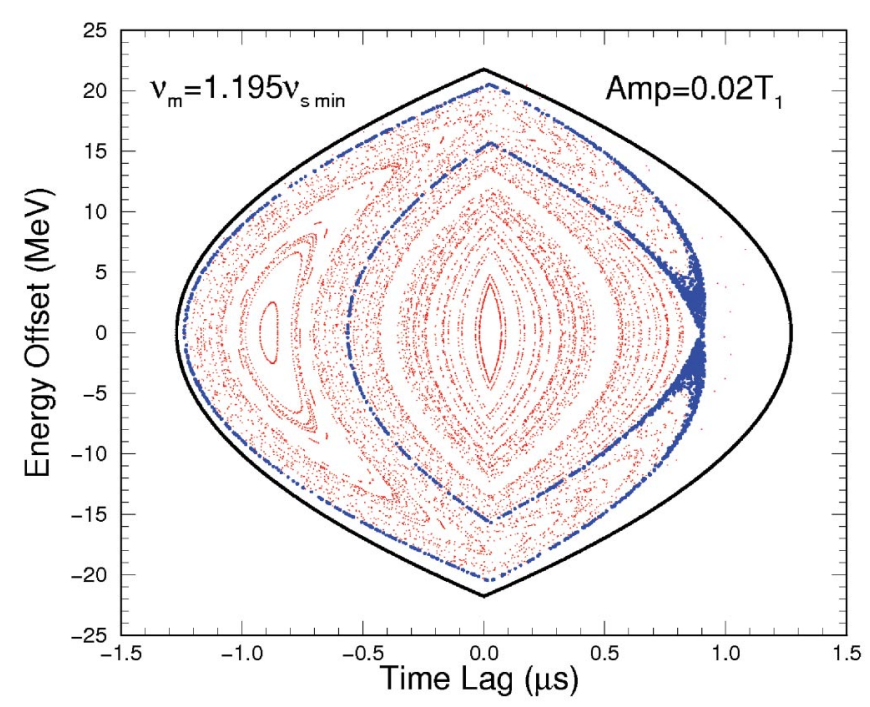

FIG. 7. (Color) Poincaré section showing the excitation of the $1: 1$ resonance by rf phase modulation at $\nu_{m}=1.195 \nu_{s, \min }$ and modulation amplitude $a T_{1}=0.02 T_{1}$. The separatrices in thicker curves show an unstable fixed point on the right and a stable fixed point on the left. Since the separatrices almost touch the barrier bucket boundary, particles outside the separatrices will be lost. The simulation involves initially 100 randomly but uniformly distributed particles tracked for $5 \times 10^{6}$ revolution turns. 
of $m: 1$ resonant islands consists of $m$ islands. There are $m$ stable fixed points and $m$ unstable fixed points equally spaced in a circle of radius $(-J)^{1 / 3}=2 m p /\left(3 \nu_{m}\right)$. With $a>0$, the stable fixed point of the 1:1 resonance is at $\psi=$ $+\pi / 2$ (the negative $\tau$ axis) while the unstable fixed point is at $\psi=-\pi / 2$ (the positive $\tau$ axis), or

$$
\tau=\mp\left(\frac{m \nu_{s, \min }}{\nu_{m}}\right)^{2} T_{1} \quad \text { and } \quad \Delta E=0 .
$$

The simulation in Fig. 7 with $\nu_{m}=1.195 \nu_{s, \min }$ shows the 1:1 resonance, with the stable and unstable fixed points at $\tau= \pm 0.89 \mu \mathrm{s}$. Graphically, they can be solved exactly by plotting $\nu_{s}$ as a function of $W$ and drawing a line $\nu_{m}=$ $1.195 \nu_{s}$. Where the line and the curve meet determines the bifurcated stable and unstable fixed points. Although the separatrices do not actually touch the outside edge of the barrier bucket, particles outside the separatrices will probably be lost. It is interesting to note that the positions of the $m: 1$ resonance fixed points do not depend on the modulation amplitude $a$. However, the island width or the separation of the intercepts of the separatrix at the positive timeadvance axis does increase with the modulation amplitude. Let us write $m=2 n+1$. For even/odd $n$, one unstable fixed point is on the positive/negative $\tau$ axis while the resonant island intercepts the negative/positive $\tau$ axis twice. To derive these intercepts, we require the equation describing the separatrices, which can be obtained by equating the Hamiltonian in Eq. (4.7) to the Hamiltonian value evaluated at the unstable fixed point on the positive/ negative $\tau$ axis when $n$ is even/odd and set $\psi=(-1)^{n} \frac{\pi}{2}$ for the intercepts at the negative/positive $\tau$ axis. Thus

$$
-p(-J)^{2 / 3}-\frac{\nu_{m}}{m} J-\frac{a e V_{0} T_{1}}{m \pi^{2}}=-\frac{4 m^{2} p^{3}}{27 \nu_{m}^{2}}+\frac{a e V_{0} T_{1}}{m \pi^{2}},
$$

which is $n$-independent. Introducing the parameters

$$
x=\frac{3 \nu_{m}(-J)^{1 / 3}}{m p}, \quad \alpha=\sqrt{1-A}, \quad A=\frac{12 a \nu_{m}^{2}}{\pi m^{3} \nu_{s, \text { min }}^{2}},
$$

the intercepts are now given by the solution of the cubic equation

$$
x^{3}-3 x^{2}+4 \alpha^{2}=0,
$$

which can be rewritten as

$$
3\left(\frac{\alpha}{x}\right)-4\left(\frac{\alpha}{x}\right)^{3}=\alpha
$$

The solution can now be easily written down by identifying $\alpha / x$ with $\sin \phi$, or

$$
x=\frac{\alpha}{\sin \phi} \quad \text { with } \phi=\left\{\begin{array}{l}
\frac{1}{3} \sin ^{-1} \alpha \\
\frac{1}{3} \sin ^{-1} \alpha+\frac{2 \pi}{3} \\
\frac{1}{3} \sin ^{-1} \alpha+\frac{4 \pi}{3}
\end{array}\right.
$$

The third solution is unphysical because it leads to $(-J)^{1 / 3}<0$. When $A \ll 1$, the approximate intercepts of the two edges of the resonant island are

$$
(-J)^{2 / 3}=\left(\frac{2 m p}{3 \nu_{m}}\right)^{2}\left[1 \pm \sqrt{\frac{4|A|}{3}}+\frac{A}{9}+\mathcal{O}\left(A^{3 / 2}\right)\right] .
$$

For the parameters of the 1:1 resonance used in the simulation, the first and second solutions correspond to $\tau=1.24$ and $0.594 \mu \mathrm{s}$, which are, respectively, the outer and inner intercepts. The third solution gives $x \sim$ $(-J)^{1 / 3}<0$, which is unphysical. Thus to avoid beam loss, particle injected into this bucket should not exceed $|\tau| \approx 0.89 \mu \mathrm{s}$. Even if this criterion is satisfied, some particles situated near $|\tau| \sim 0.594 \mu \mathrm{s}$ and $\Delta E=0$ where one separatrix is situated will follow the separatrix to the chaotic region near the unstable fixed point and will be lost eventually. Some particles will oscillate around the bifurcated stable fixed point, landing near the boundary of the barrier bucket, with the result of lengthening the beam. To avoid significant bunch lengthening, the initial half-bunch length must be $|\hat{\tau}| \lesssim 0.594 \mu \mathrm{s}$ under this particular modulation.

We next want to investigate whether or not adjacent chains of resonant islands will overlap each other when many parametric resonances are excited under a large modulation tune $\nu_{m}$. The separation between the $m: 1$ and $m+2: 1$ is given by

$\Delta(-J)^{2 / 3}=\left(\frac{2 p}{3 \nu_{m}}\right)^{2}\left[(m+2)^{2}-m^{2}\right]=4(m+1)\left(\frac{2 p}{3 \nu_{m}}\right)^{2}$,

while the half-widths of the resonance islands are from Eq. (4.16)

$$
\delta(-J)^{2 / 3}=\sqrt{\frac{4|A|}{3}} m^{2}\left(\frac{2 p}{3 \nu_{m}}\right)^{2} .
$$

The condition for no overlapping is $\delta J^{2 / 3}<\Delta J^{2 / 3}$, or

$$
|A|<12\left(\frac{m+1}{m^{2}}\right)^{2}
$$

Unlike the situation of voltage modulation, the parameter $A$ here is given by the complicated expression in Eq. (4.12), which is proportional to $\nu_{m}^{2}$. Thus for no island overlapping, the modulation amplitude must satisfy

$$
|a|<\frac{8 \pi^{2}}{9} \frac{(m+1)^{2}}{m \nu_{m}^{2}} \frac{p^{3}}{e V_{0} T_{1}}=\frac{\pi(m+1)^{2}}{m\left(\nu_{m} / \nu_{s, \min }\right)^{2}} .
$$

It will be the lower parametric resonances that are mostly easily overlapped. Thus we set $m=1$ and obtain the nooverlap criterion

$$
|a|<\frac{12.6}{\left(\nu_{m} / \nu_{s, \min }\right)^{2}} .
$$


For example, overlapping will occur when $\nu_{m} \gtrsim 25 \nu_{s, \min }$ at the modulation amplitude of $a=0.02$. When overlapping of island chains occurs, the chaotic region first exists close to the center of the bucket and extends outward as the modulation tune and modulation amplitude increase. Figure 8 shows a simulation result of phase modulation with modulation amplitude $a=0.02$ and modulation tune $\nu_{m}=29.08 \nu_{s, \text { min }}$ corresponding to the modulation frequency of $60 \mathrm{~Hz}$. The blue dots show the Poincaré section of one particle initially at $\Delta E=0$ and $\tau=-0.035 \mu \mathrm{s}$ streaming outwards until $|\tau|<0.31 \mu \mathrm{s}$. This demonstrates the overlapping of resonance islands up to the 15:1 resonances in rough agreement with the estimation derived above. When the modulation frequency is increased to $120 \mathrm{~Hz}\left(\nu_{m}=58.16 \nu_{s, \min }\right)$, the chaotic region extends to $|\tau| \sim 0.8 \mu$ s or the $47: 1$ resonant islands. The chaotic regions for these two one-particle simulations are shown separately in the top plot of Fig. 9. Upon further increasing the modulation frequency to, for example, $180 \mathrm{~Hz}\left(\nu_{m}=\right.$ $\left.87.24 \nu_{s, \min }\right)$, the chaotic region in the bucket becomes larger than the whole bucket according to our derivation. This is demonstrated by the one-particle Poincare section depicted in the lower plot of Fig. 9, where the particle initially at $\tau=-0.2265 \mu \mathrm{s}$ and $\Delta E=0$. We see that the particle diffuses outwards and leaves the bucket after 7057 modulation periods or $3.52 \times 10^{6}$ revolution turns. The initial position of the particle is extremely critical to having the particle diffused outwards. For example, if the particle starts with $\tau=-0.2260 \mu \mathrm{s}$ and $\Delta E=0$ instead, it dif-

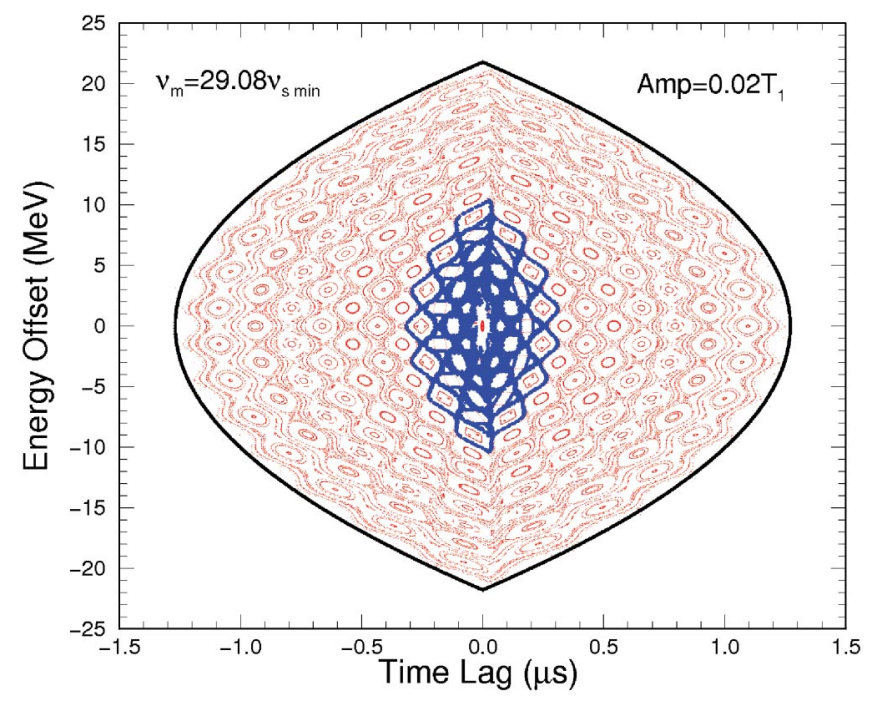

FIG. 8. (Color) Poincaré section showing the phase modulation with amplitude $a T_{1}=0.02 T_{1}$ and modulation tune $\nu_{m}=$ $29.08 \nu_{s, \min }$ (or modulation frequency $60 \mathrm{~Hz}$ ). The simulations was performed with 50 particles equally spaced on the timeadvance axis and tracking for $5 \times 10^{6}$ turns. Particles clustered around the center of the bucket (blue dots) $|\tau| \leq 0.035 \mu$ s and $\Delta E=0$ are seen to diffuse towards $|\tau| \sim 0.31 \mu \mathrm{s}$, indicating a sea of chaos. fuses out to $|\tau| \sim 0.83 \mu$ s only after $10 \times 10^{6}$ turns. With $\tau=-0.2270 \mu \mathrm{s}$ initially, the particle goes out only to $|\tau| \sim 0.61 \mu \mathrm{s}$ and goes inwards to $|\tau| \sim 0.13 \mu \mathrm{s}$ after $10 \times 10^{6}$ turns. Continuing the tracking up to $100 \times 10^{6}$ turns does not appear to change the results. We do not quite understand the reason behind this. We suspect that particles diffuse very slowly in the region when $W \leqq 0.25 \mu$ s where there are many overlapping resonances. There is a good chance for a particle's starting position be very near to one of the stable fixed points and no diffusion takes place afterwards. The particle can also be very close to an unstable fixed point where the particle movement becomes extremely slow. It is possible that a very very large number of modulation periods will be required to see the movement of these particles.

To estimate the tolerance of the rf phase modulation, we calculate the maximum stable bunch area of the rf system. We randomly and uniformly populate 5000 particles inside the bucket area and track the beam for more than 100 synchrotron oscillations (with reference to $\nu_{s, \min }$ ). The fractional stable area in unit of the bucket area is defined as the ratio between the number of survival particles and the number of initial particles. The result is depicted in Fig. 10 as functions of modulation tune. The traces from the top downwards correspond to fractional modulation amplitude $a=0.0001,0.001,0.005,0.01,0.02,0.03$, $0.04,0.05,0.06,0.07,0.08,0.09$, and 0.1 . At small modulation amplitudes, we see particle loss peak when the modulation tunes $\nu_{m}$ are odd multiples of $\nu_{s, \min }$ and decreases as the modulation tune increases as predicted by theory. As the modulation amplitude increases, the loss peaks move to higher modulation tunes. This is also understandable because the bifurcated stable and unstable fixed points have to move somewhat inwards from the bucket boundary in order to move more particles from the beam to outside the barrier bucket. These loss peaks are broadened as the modulation amplitude increases and even try to merge into each other.

We demonstrated above that a chaotic sea will form in the barrier bucket when the modulation tune $\nu_{m}$ or the modulation amplitude $a$ is large enough. Thus Fig. 10 may be not showing the steady-state survival fractions. For this reason, we repeat the simulations for every point for a duration 10 times longer, up to 1000 synchrotron oscillations (with reference to $\nu_{s, \min }$ ), or $8.1 \mathrm{~min}$. The result is shown in Fig. 11. Although the resonance strength has a $m^{-1}$ dependency, we see the tendency that more particles are leaving the barrier bucket as the modulation tune increases to $\gtrsim 10 \nu_{s, \min }$. Obviously, this is because of the development of stochasticity as adjacent chains of island overlap. For example, the overlap criterion of Eq. (4.21) shows that at $a=0.1$, overlapping occurs when $\nu_{m} / \nu_{s, \min } \gtrsim 11$. Comparing with Fig. 6 , it is obvious that rf phase modulation is more devastating than rf voltage modulation. 

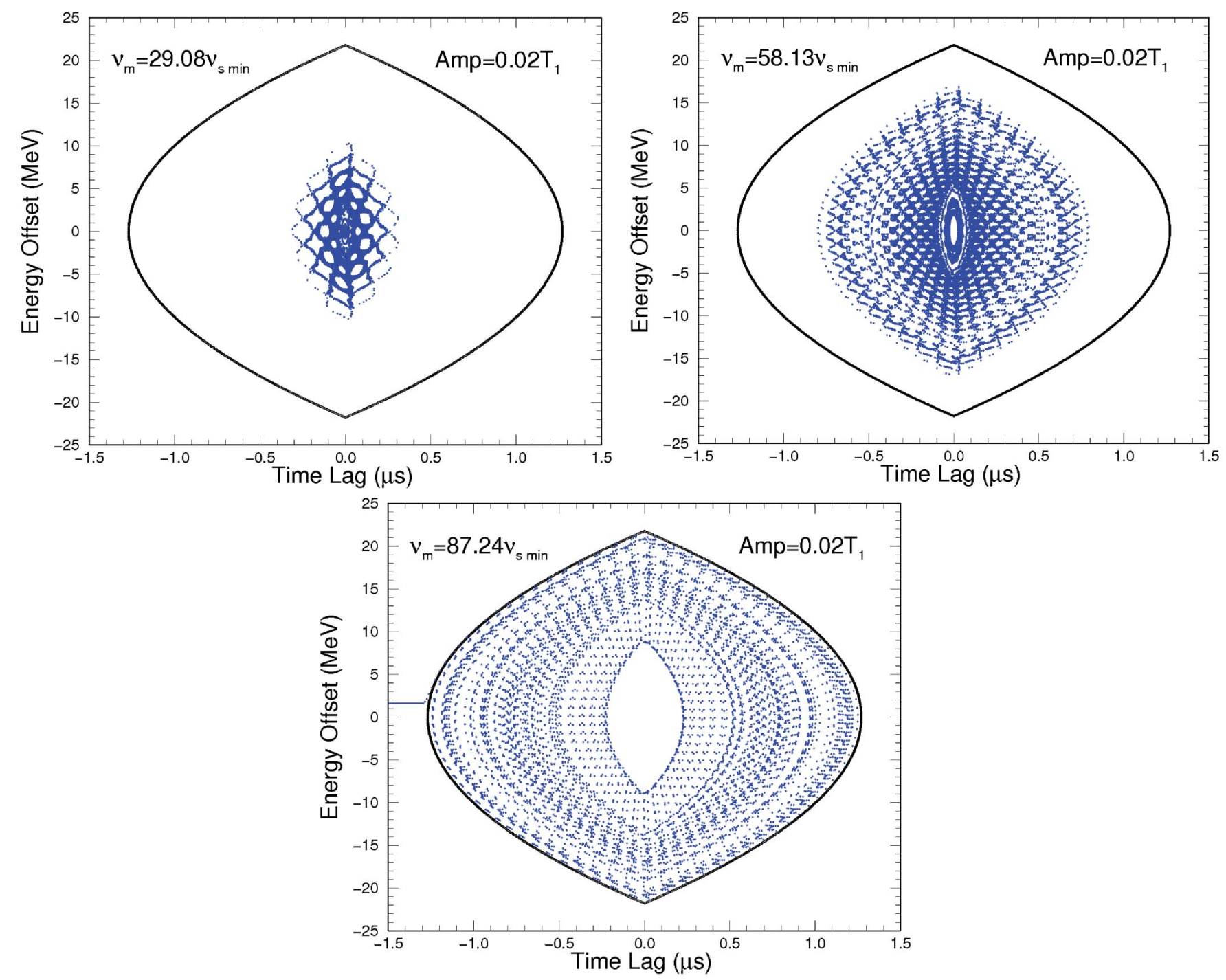

FIG. 9. (Color) Poincaré sections showing chaotic regions when the rf phase modulation tunes are, respectively, $\nu_{m}=29.08 \nu_{s, \text { min }}$ (top left), $\nu_{m}=58.13 \nu_{s, \min }$ (top right), and $\nu_{m}=87.24 \nu_{s, \min }$ (lower) (or modulation frequencies 60, 120, and 180 Hz). The phase modulation amplitude is $a T_{1}=0.02 T_{1}$ in all 3 cases. Each plot is made by tracking one particle initially at $\Delta E=0$ and $\tau=$ $-0.035 \mu \mathrm{s}$ (top left), $\tau=-0.08 \mu \mathrm{s}$ (top right), and $\tau=-0.2265 \mu \mathrm{s}$ (lower). The top two plots are tracked for $10 \times 10^{6}$ turns. In the lower plot, the particle finally leaves the bucket after 7057 modulation periods or $3.52 \times 10^{6}$ revolution turns.

\section{SYNCHROBETATRON COUPLING}

Coupled motion between the transverse and longitudinal degrees of freedom is called synchrobetatron coupling. The synchrobetatron coupling is important to electron storage rings, where the fractional parts of the synchrotron and betatron tunes are of the same order of magnitude. On the other hand, the fractional parts of the betatron and synchrotron tunes differ substantially in hadron storage rings, and the coupling between the longitudinal and transverse motions becomes less important. However, it has been demonstrated at the IUCF Cooler Ring that slow modulation of a dipole can excite synchrobetatron coupling in a proton beam. [6]
An extra dipole field will lead to an extra bending of the beam particle by the angle $\Delta \theta$. This will result in lengthening the closed orbit of the beam particle by $\Delta C=D \Delta \theta$, where $D$ is the dispersion at the location of this extra dipole field. Thus the beam particle will arrive at the rf cavity with a phase error $\Delta T=\Delta C T_{0} / C$ in time, where $C$ is the circumferential length of the closed orbit. If this extra dipole field is the result of a modulation with tune $\nu_{m}$, which is of the order of the synchrotron tune and is small, this rf phase error will accumulate for roughly half the modulation period before deaccumulation takes place. Suppose that the rf phase error behaves as $\Delta T \cos 2 \pi n \nu_{m}$ at the $n$th revolution turns, the accumulated rf phase error from the first to the $n$th turn is 


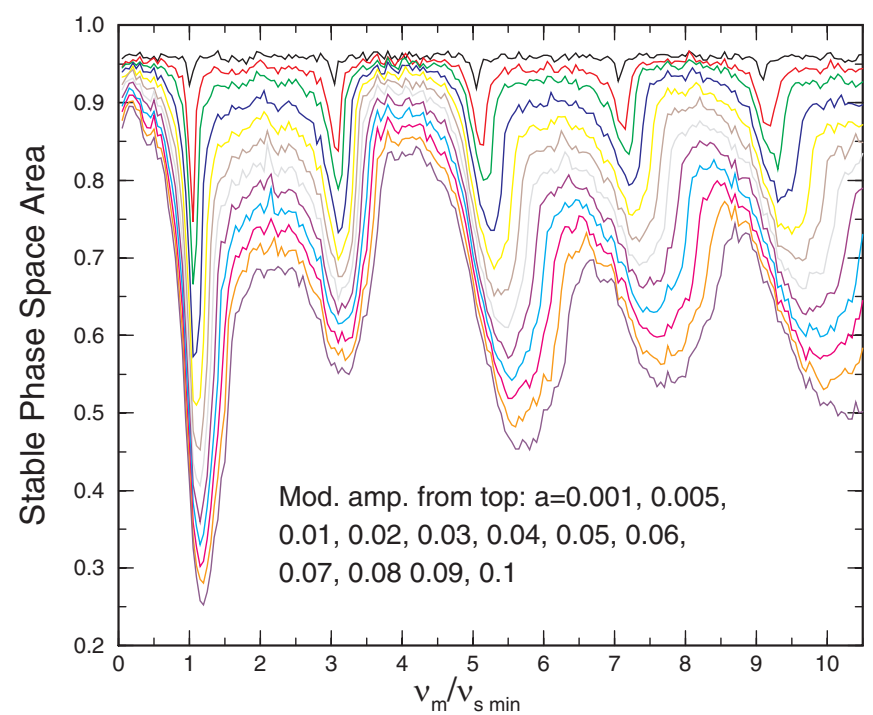

FIG. 10. (Color) Fractional stable bunch area in ratio to the bucket area as functions of phase modulation tune, for fractional modulation amplitudes, from top to bottom, $a=0.0001,0.001$, $0.005,0.01,0.02,0.03,0.04,0.05,0.06,0.07,0.08,0.09,0.1$. Parametric resonances at $m: 1$ with $m=1,3,5, \cdots$ are evident, although their widths become very much broadened as $m$ increases. Among them, the 1:1 resonance is the most devastating. The simulation for each point was performed for the duration of 100 synchrotron periods, with respect to $\nu_{s, \min }$, about $48.6 \mathrm{~s}$.

$$
\Delta T_{\mathrm{acc}}=\Delta T \int_{0}^{n} \cos 2 \pi \nu_{m} n \Delta n
$$

The maximum accumulation takes place when the duration of the modulation phase becomes $\phi_{m}=2 \pi \nu_{m} n=\pi / 2$, and the above integral gives an accumulation enhancement factor of $1 /\left(2 \pi \nu_{m}\right)$. This is shown schematically in Fig. 12. Thus the rf phase error will oscillate at the modulation tune with the amplitude

$$
a T_{1}=\Delta T_{\mathrm{acc}}=\frac{D \Delta \theta}{2 \pi \nu_{m} C} T_{0} .
$$

If we wish to fill the barrier bucket up to $92 \%$, we learn from simulation that the rf phase error has to be less than $a \sim 0.0001$ (not shown in Fig. 11). This implies that the allowable orbit lengthening is

$$
\Delta C=D \Delta \theta=\frac{2 \pi \nu_{m} a C T_{1}}{T_{0}}=5.47 \times 10^{-6} \mathrm{~m},
$$

where we have used the circumferential length of the Recycler Ring $C=3319 \mathrm{~m}$, revolution period $T_{0}=$ $11.13 \mu \mathrm{s}$, and $\nu_{m}=\nu_{s, \min }=2.297 \times 10^{-5}$.

Consider a typical quadrupole set in the Recycle Ring. It consists of two half-quadrupoles each of length $\Delta \ell / 2=$ $0.508 \mathrm{~m}$ (20 in) separated by $1 \mathrm{~m}$ and a normalized field gradient $K_{1}=B_{y}^{\prime} /(B \rho)=0.0886 \mathrm{~m}^{-2}$, where $(B \rho)$ is the rigidity of the beam. The lattice of the Recycler Ring is essentially FODO in structure - one consisting of alternating focusing and defocusing lenses. The half FODO cell is

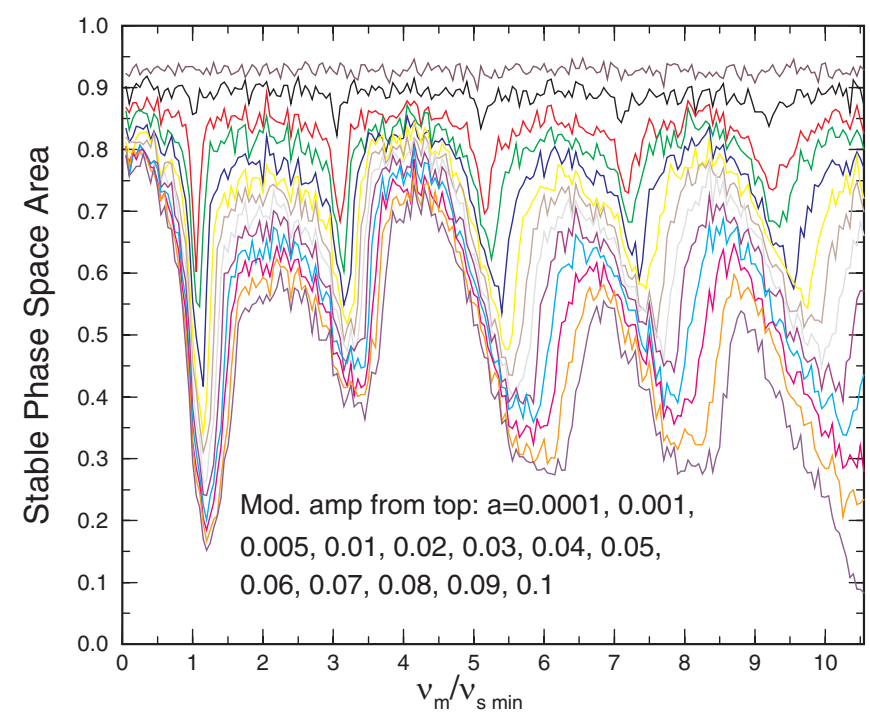

FIG. 11. (Color) Same as Fig. 10, but the simulation duration for every point has been increased 10 times, to 1000 synchrotron periods, with respect to $\nu_{s, \min }$, about $486 \mathrm{~s}$ or $8.1 \mathrm{~min}$. The chaotic behavior becomes more apparent. Note that the vertical scale is different from that of Fig. 10.

$17.28 \mathrm{~m}$ long. If the quadrupole set has a horizontal offset of $\Delta x$ from the designed orbit, the beam will see an extra dipole field

$$
\Delta B_{y}=K_{1} \Delta x(B \rho)
$$

After traversing the quadrupole complex, the beam will receive an extra bend of

$$
\Delta \theta=\frac{\Delta B_{y} \Delta \ell}{(B \rho)}=K_{1} \Delta x \Delta \ell .
$$

For a displacement of $\Delta x=1 \mu \mathrm{m}$, this offset quadrupole complex will result in the lengthening of the closed orbit by

$$
\Delta C=D \Delta \theta=0.200 \times 10^{-6} \mathrm{~m} \text {, }
$$

where the maximum dispersion of $D=2.227 \mathrm{~m}$ at the Fquadrupoles has been used. This lengthening is about 27.4 times less than stability criterion derived in Eq. (5.3).

There are many possibilities of exciting vibration in the Recycler quadrupoles. One is ground motion. Suppose that a truck is driven on the service road about $22 \mathrm{~m}$ above the Recycler Ring. If horizontal vibrations are excited in 28 consecutive quadrupole sets with an amplitude of $\Delta x=$ $1 \mu \mathrm{m}$ at the modulation tune $\nu_{s, \min }$, the stability criterion will be surpassed. The next possibility is the influence of the low conducting water (LCW) piping system of the Main Injector which shares the same tunnel with the Recycler Ring. The LCW pipe support is connected to the same channel in the concrete as the stands of the Recycler magnets, so that vibrations in the LCW system can be transported to the Recycler magnets. There are 104 FODO cells or 208 sets of quadrupoles. If they oscillate randomly, the lengthening of the closed orbit will be 


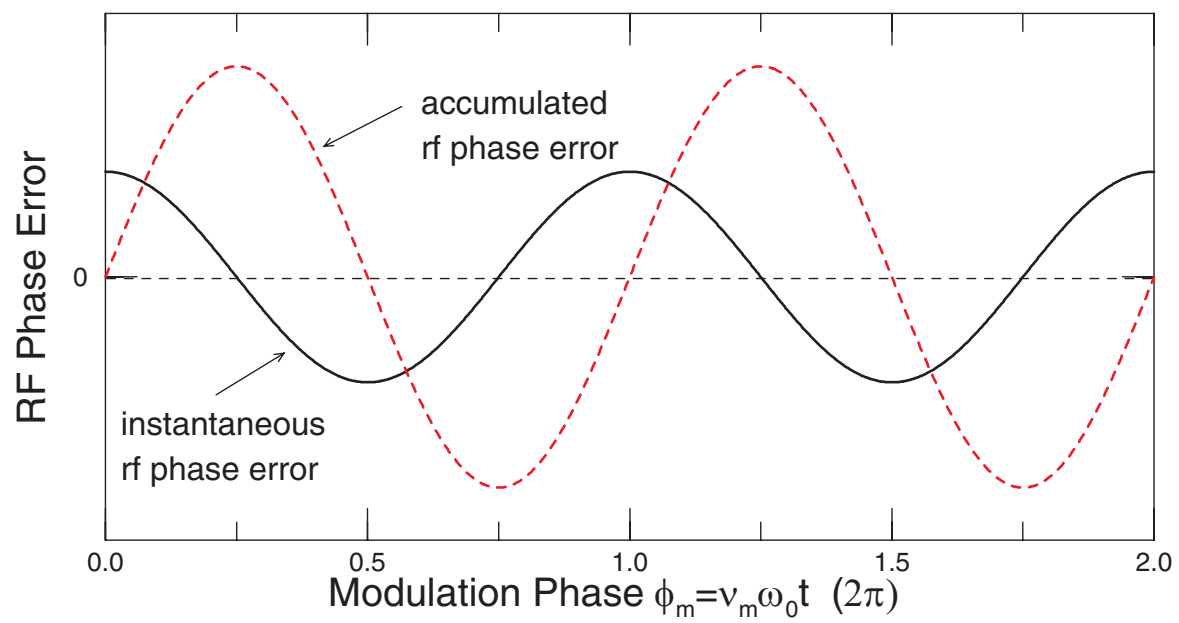

FIG. 12. (Color) Instantaneous rf phase error and the accumulated rf phase error as functions of modulation phase $\phi_{m}=\nu_{m} \omega_{0} t$.

enhanced $\sqrt{208}=14.4$ times that of one quadrupole set. Here, we also need to remember that the stability criterion will be increased when the modulation tune $\nu_{m}$ is larger than $\nu_{s, \min }$, which is $2.297 \times 10^{-5}$ or $2.06 \mathrm{~Hz}$. The natural frequency of horizontal vibration of the Recycler magnet was found to be $9.6 \mathrm{~Hz}$, [7] thus pushing up the stability criterion by a factor of 4.7. As a result, an oscillation amplitude of $\Delta x \approx 9 \mu \mathrm{m}$ will surpass the stability criterion. Another possibility is the $60 \mathrm{~Hz}$ electrical noise corresponding to the modulation tune $\nu_{m}=29.08 \nu_{s, \min }$. If all the quadrupole sets are excited randomly, an amplitude of oscillation of $\Delta x \approx 55 \mu \mathrm{m}$ is required to surpass the stability criterion.

\section{CONCLUSIONS}

(1) We have studied the stability of a barrier bucket under the voltage or $r f$ phase modulation when the separation of the two barriers is $T_{2}=0$. We find that the consequence of phase modulation is more severe than voltage modulation.

(2) The synchrotron tune for such a barrier is infinite when the barrier penetration $W=0$. It then decreases as $W^{-1 / 2}$ to a minimum $\nu_{s, \min }$ when $W=T_{1}$, the width of the barrier. A modulation tune larger than $m \nu_{s, \min }$ will excite all parametric $n: 1$ resonances with $n=2,4,6, \cdots$, up to the largest even integer below $m$ under voltage modulation, and parametric $n: 1$ resonances $1,3,5, \cdots$, up to the largest odd integer below $m$ under phase modulation. We have shown both mathematically and through simulations that the even resonances under voltage modulation will not overlap when the modulation amplitude $a<1$. However, the odd resonances under phase modulation will overlap even for small modulation amplitude if the modulation tune is large enough. The implication is that a small bunch at the center of the bunch may diffuse outward with a large increase in bunch area and possible beam loss under rf phase modulation.
(3) With the aid of simulations, we have determined the modulation amplitude to safeguard a certain fraction of the barrier bucket against voltage and phase modulation instabilities. For example, to safeguard $95 \%$ of the barrier bucket, the voltage modulation amplitude should be less than $a V_{0}=0.001 V_{0}$. To safeguard $92 \%$ of the barrier bucket, the rf phase modulation amplitude should be less than $a T_{1}=0.001 T_{1}$ for modulation up to $\sim 1 \mathrm{~min}$ and $a T_{1}=0.0001 T_{1}$ for modulation up to $\sim 8 \mathrm{~min}$.

(4) We have analyzed synchrobetatron coupling in the Recycler Ring. If the horizontal vibration amplitudes of the Recycler gradient magnets are large, synchrobetatron coupled motion will be excited, and some portion of the barrier bucket will become unstable.

(5) When there is a small separation between the two confining barriers, the situation is not improved. Figure 13 plots the synchrotron tune as functions of energy offset on the left and barrier penetration $W$ on the right, when the barrier separation $T_{2}$ equal to $T_{1}$, the width of the barriers. In the same figure, we plot in dashes also $2 \nu_{s}, 3 \nu_{s}, \cdots$. We see that the synchrotron tune increases linearly in $\Delta E$ at the center of the bucket, reaches a maximum and rolls off slowly as $1 / \Delta E$. This makes us recall the similar synchrotron tune behavior for a Landau cavity system. The dependence on barrier penetration is $\sqrt{W}$ starting from $W=0$, and rolls off as $1 / \sqrt{W}$ after reaching a peak. The peak value of the synchrotron tune is given by

$$
\nu_{s, \max }=\frac{|\eta| e V_{0} T_{0}}{32 \beta^{2} E_{0} T_{2}} .
$$

Since we have chosen $T_{2}=T_{1}$, this happens to be exactly the same as our former $\nu_{s, \text { min }}$ with zero barrier separation. As a function of maximum energy offset, the maximum is located at

$$
\widehat{\Delta E}=\sqrt{\frac{\beta^{2} E_{0} e V_{0} T_{2}}{2|\eta| T_{0}}} .
$$



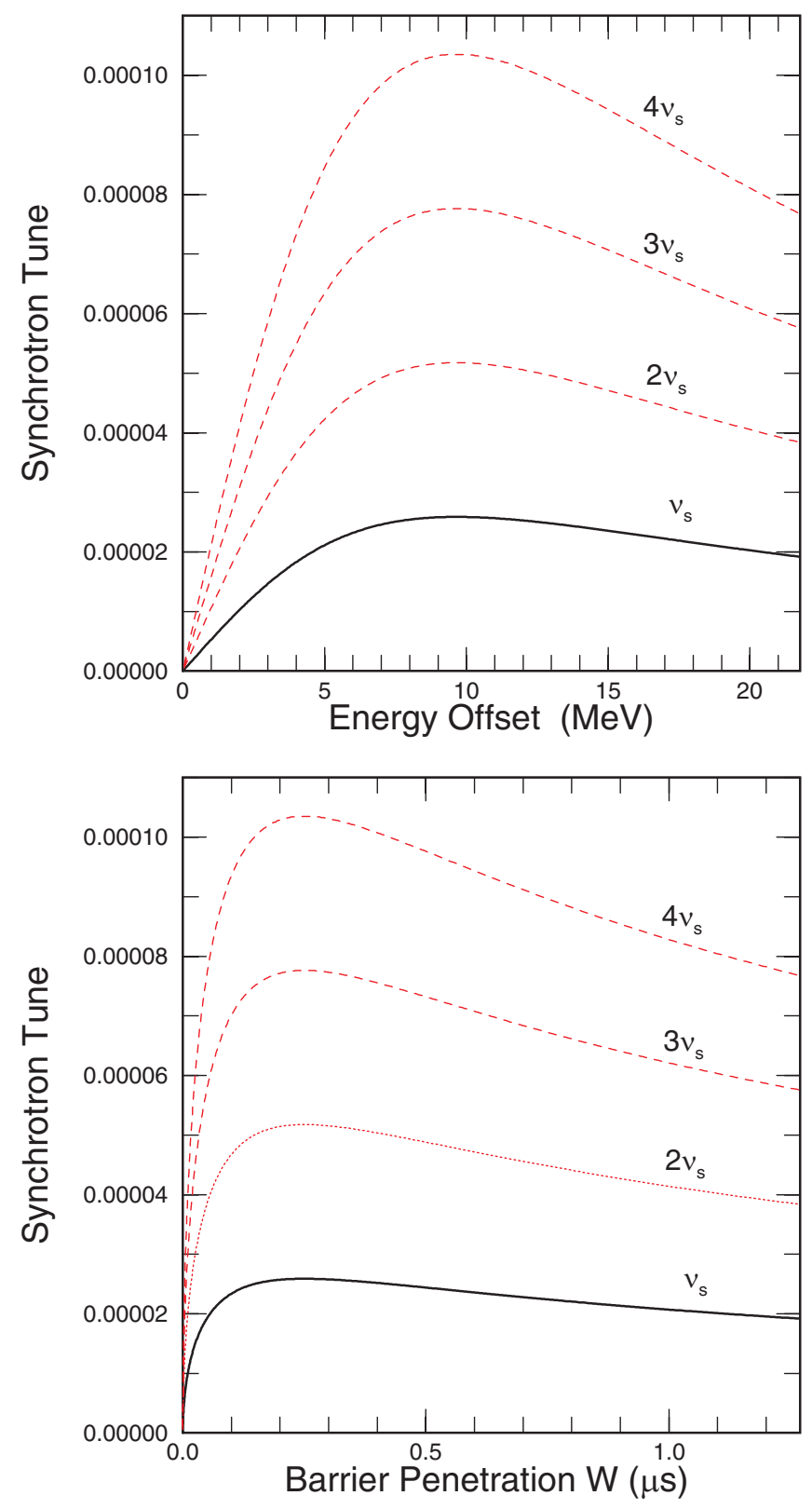

FIG. 13. (Color) Synchrotron tune as functions of energy offset on the left and barrier penetration on the right, when the barrier separation and barrier width are equal or $T_{2}=T_{1}=1.27 \mu \mathrm{s}$ and barrier voltage $V_{0}=2 \mathrm{kV}$. Also shown in the plots are higher synchrotron harmonics.

Since the peak energy offset $E_{\mathrm{pk}}$ given by Eq. (1.1) is independent of the barrier separation $T_{2}$, we see that the location of maximum synchrotron tune increases linearly with $T_{2}$ and moves towards the edge of the bucket when $T_{2}=4 T_{1}$. After that the synchrotron tune becomes monotonic. Because of the occurrence of the peak when $T_{2}<$ $4 T_{1}$, two sets of $m: 1$ resonances are created and the bifurcation takes place at the position of maximum synchrotron tune. Thus any modulation tune $\nu_{m}$ that is less than the maximum of $m \nu_{s}$ will excite all parametric $n: 1$ resonances with $n$ any even or odd integer larger than $m$, depending on whether it is voltage modulation or rf phase modulation. This is slightly different from the situation when there is no separation between the barriers $\left(T_{2}=0\right)$. Formerly, if we count from the center of the barrier bucket, we first see the 1:1 resonance under rf phase modulation, then $3: 1,5: 1, \cdots$ with the highest one near the boundary of the bucket. Here the ordering is in the reverse direction, with the higher resonances closest to the center of the bucket and the order of resonances decreases until the position of maximum synchrotron oscillation amplitude is reached. This implies more resonances are excited, resulting in more overlapping of island chains and more chaotic region near the center. Simulations show that there is a sea of chaos near the center of the bucket under rf phase modulation, but such sea of chaos does not show up with voltage modulation.

[1] For finite separation between the inner edge of the two barriers but with the separation less than 4 times the barrier width $\left(T_{2}<4 T_{1}\right)$, the synchrotron tune increases linearly from zero at zero energy offset and goes through a maximum $\nu_{s, \max }=\sqrt{|\eta| e V_{0} T_{0} /\left(32 \beta^{2} E_{0} T_{2}\right)}$ before decreasing again [2]. When $T_{2}=0$, however, the synchrotron tune decreases monotonically from infinity for trajectories with zero to maximum barrier penetration.

[2] S. Y. Lee and K. Y. Ng, Phys. Rev. E 55, 5992 (1997).

[3] The sign on the right side of Eq. (2.1) has been so chosen that a particle with positive energy offset $(\Delta E>0)$ will arrive earlier in the next revolutionary turn below transition $(\eta<0)$.

[4] If we choose the starting point of $\psi=0$ at $\tau=-W$ and $\Delta E=0$ or $\tau=+W$ and $\Delta E=0$, the new resonant strength functions $F_{2 n}^{\text {new }}$ will defer from the old one $F_{m}(J)$ by a phase that is dependent on $m$. This comes about because the expansion of $f_{0}\left(\tau, T_{1}\right)$ is now in terms of the new angle variable $\psi_{\text {new }}$, which is related to the old one by $\psi_{\text {new }}=\psi \mp \frac{\pi}{2}$. For the Hamiltonian, this amounts to a shift of $\frac{\pi}{2}$ in $\mp \psi$ in Eq. (3.6) below.

[5] We compare the half but not the full island width with the island-chain separation, because two consecutive chains of islands are intertwined with each other; i.e., the stable fixed points of one chain have roughly the same $\psi$-angle values as the unstable fixed points of the adjacent chain. For the criterion of Eq. (3.27), the island chain labeled by $n$ has stable fixed points on the $\tau$ axis while the adjacent one labeled by $n+1$ has unstable fixed points on the $\tau$ axis.

[6] M. Syphers, M. Ball, B. Brabson, J. Budnick, D. D. Caussyn, A. W. Chao, J. Collins, V. Derenchuk, S. Dutt, G. East, M. Ellison, T. Ellison, D. Friesel, W. Gabella, B. Hamilton, H. Huang, W. P. Jones, S. Y. Lee, D. Li, M. G. Minty, S. Nagaitsev, K. Y. Ng, X. Pei, G. Rondeau, T. Sloan, L. Teng, S. Tepikian, Y. Wang, Y. T. Yang, and P. L. Zhang, Phys. Rev. Lett. 71, 719 (1993).

[7] G. Jackson and C. Gattuso, Fermilab Main Injector Notes No. MI-0235, 1998; http://beamdocs.fnal.gov/DocDB/ 0003/000326/001/MI-0235.pdf. 